\title{
Superconducting mesa-structures with a spin filtering manganite interlayer
}

\author{
Karen Constantinian*, Gennady Ovsyannikov, Yulii Kislinskii, Victor Demidov, \\ Anton Shadrin, Andrey Petrzhik
}

Kotel'nikov Institute of Radio Engineering and Electronics RAS, Moscow, Russia

\begin{abstract}
We report on current transport and magnetism in hybrid superconducting mesa-structures prepared from epitaxial superconductor $\mathrm{YBa}_{2} \mathrm{Cu}_{3} \mathrm{O}_{7-\delta}$ and manganite $\mathrm{LaMnO}_{3}$ thin films. Superconducting $\mathrm{Au}-\mathrm{Nb}$ bilayer was the top electrode. Measurements of magnetic resonance in heterostructure with the only $\mathrm{Au}$ top electrode (without $\mathrm{Nb}$ ) showed ferromagnetic state close to Curie temperature of $\mathrm{LaMnO}_{3}, 150 \mathrm{~K}$. A spin filtering was revealed by measurements of tunneling magneto-resistance. Mesa-structures had no superconducting current even at reduced temperature $T=300 \mathrm{mK}$ and the smallest manganite interlayer thickness, $d_{M}=5.6 \mathrm{~nm}$. Varying the external magnetic field $|H|<10$ Oe and dc bias current $|I|<0.5 \mathrm{~mA}$ the microwave generation with power $P<1 \mathrm{pW}$ with a line-width of order $50 \mathrm{MHz}$ was observed in $\mathrm{GHz}$ frequency band. The frequency of generation could be tuned by the biasing current with a rate $10^{13} \mathrm{~Hz} / \mathrm{A}$. The impact of spin polarized current on microwave generation is discussed taking into account the asymmetry of oscillation amplitudes over the applied magnetic field.
\end{abstract}

\section{Introduction}

Recently, superconducting structures with a spinpolarized current have attracted increasing interest in view of the prospects for their use in spintronics and novel elements of microwave electronics. Interaction of superconductivity and magnetism was studied in hybrid structures comprised from superconductors (S) and ferromagnetic materials $(F)$, tunnel junctions with a ferromagnetic barrier $[1,2]$ and etc. Tunnel current through a ferromagnetic barrier becomes spin-polarized due to the splitting of the energy band into the spin-up and spin-down levels [3, 4]. The precession of magnetization excited by a spin-polarized current was theoretically considered in [5-7]. Experimentally, the excitation of precession of the magnetization with a sufficiently high current density (above $10^{6} \mathrm{~A} / \mathrm{cm}^{2}$ ) was observed in multilayer structures made of ferromagnets and metals [8-10] and in the tunnel-type structure [11]. At the same time, the dynamics of the spin-polarized current in superconducting structures with a ferromagnetic interlayer remains less studied.

\section{Experimental}

Hybrid mesa-structures were prepared from epitaxial films of superconducting cuprate $\mathrm{YBa}_{2} \mathrm{Cu}_{3} \mathrm{O}_{\mathrm{x}}$ (YBCO) and manganite $\mathrm{LaMnO}_{3}$ (LMO) - materials having good chemical and crystalline compatibility [12]. The top superconducting electrode was thin film bilayer consisting of $\mathrm{Nb}$ and $\mathrm{Au}$ film, providing the contact with
LMO manganite. The superconducting YBCO film was produced by laser ablation at temperature of $700-800^{\circ} \mathrm{C}$ on the substrate (110) of $\mathrm{NdGaO}_{3}$ (NGO). A thin film of LMO interlayer $d_{M}=5-20 \mathrm{~nm}$ thick was grown epitaxially in the same vacuum chamber in situ at high temperature, and after cooling to room temperature was covered with an Au layer 20-30 nm thick. The critical temperature of the YBCO film was $T_{C}=88-89 \mathrm{~K}$. The subsequent layer of $\mathrm{Nb}$ was deposited by magnetron sputtering. The Au film was used to reduce the diffusion of oxygen from manganite to $\mathrm{Nb}$. The proximity effect between $\mathrm{Nb}$ and $\mathrm{Au}$ films provided a superconducting critical temperature of the bilayer $T_{C}{ }^{*}=8.5-9 \mathrm{~K}$, close to the critical temperature of the $\mathrm{Nb}$ film $(9.2 \mathrm{~K})$. Superconducting mesa-structures with interlayer of LMO and in-plane dimensions from 10x10 to 50x50 $\mathrm{m}^{2}$ (see Fig.1) were formed by means of photolithography and plasma-chemical and ion-beam etching. According to results $[13,14]$, the LMO manganite of stoichiometric composition has the properties of an insulator and is an antiferromagnet at low temperatures. But a small change in the oxygen content leads to the appearance of ferromagnetism with the Curie temperature of order 140$150 \mathrm{~K}$. A ferromagnetism for the LMO films was reported in [15] and confirmed by measurements of temperature dependence of ferromagnetic resonance magnetic field $H_{0}(T)$ [16]. Fig.2 shows temperature dependences $\rho(T)$ of resistivity of LMO film and the ferromagnetic resonance dependence. The investigation of the microwave properties of mesa-structures was carried out using apparatus schematically shown in Fig.1. Low temperature measurements were carried out

*Corresponding author: karen@hitech.cplire.ru 
using cooled HEMT amplifier operating in frequency band $1-2 \mathrm{GHz}$ with the noise temperature $T_{N I}=8 \pm 2 \mathrm{~K}$ and gain $K_{l}=20 \mathrm{~dB}$ at $T=4.2 \mathrm{~K}$. The balance circuit of the input stage of the amplifier provided stable operation in a wide range of load resistance $10-100 \Omega$.

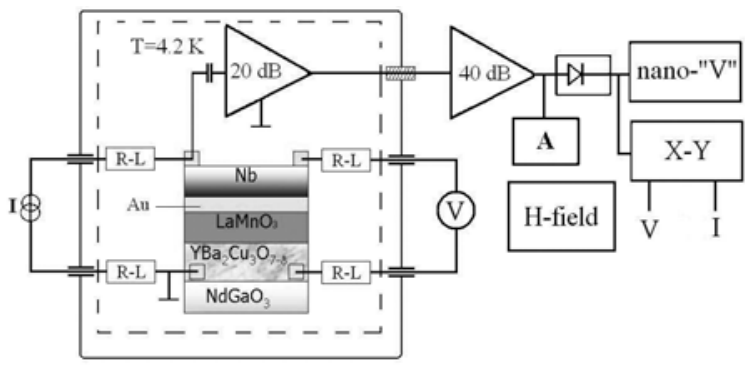

Fig.1. Experimental set-up for microwave measurements. The mesa-structure (the cross section is shown in the middle of the figure) and the cold amplifier were placed inside the magnetic field coil (dashed contour) and the screen (solid-line contour) at $T=4.2 \mathrm{~K}$. The (H-field) coil had bipolar dc feed. The dc biasing of mesa-structure (I) and voltage measurement unit (V) as well the power supply of the cold amplifier were set through the filtering (R-L) circuits and through the built-in capacitors. The amplified microwave signal from mesa-structure was monitored either by the spectrum analyzer (A), or the quadratic detector. The output signal level was registered by the nanovoltmeter (nano-"V") and recorded by (X-Y) plotter.

The noise temperature of the "room" amplifier was $T_{N 2}=130 \mathrm{~K}$ and gain $K_{2}=40 \mathrm{~dB}$. The voltage signal, proportional to the power $P$ of microwaves emitted by mesa-structure, was taken from the output of the quadratic detector. Simultaneously, the spectrum analyzer monitored the amplitude-frequency response at the output of the "room" amplifier. The magnetic field at microwave measurements was set perpendicular to the plane of the substrate and, according to the calculations [17], had efficiency close to the parallel field for the case of a sandwich tunnel junction with superconducting electrodes with a square shape.

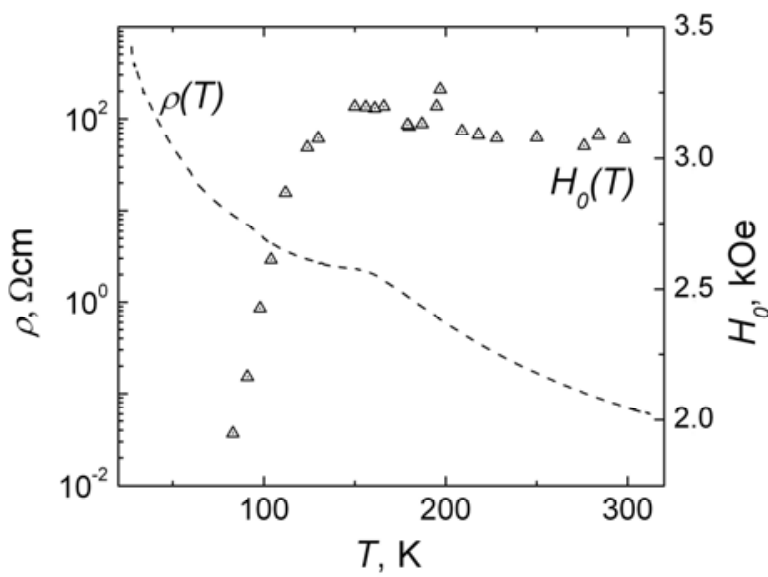

Fig.2. Temperature dependence $\rho(T)$ of resistivity of an autonomous LMO film (dashed curve). Temperature dependence of the magnetic resonance field $H_{0}$ for the LMO film (triangles).

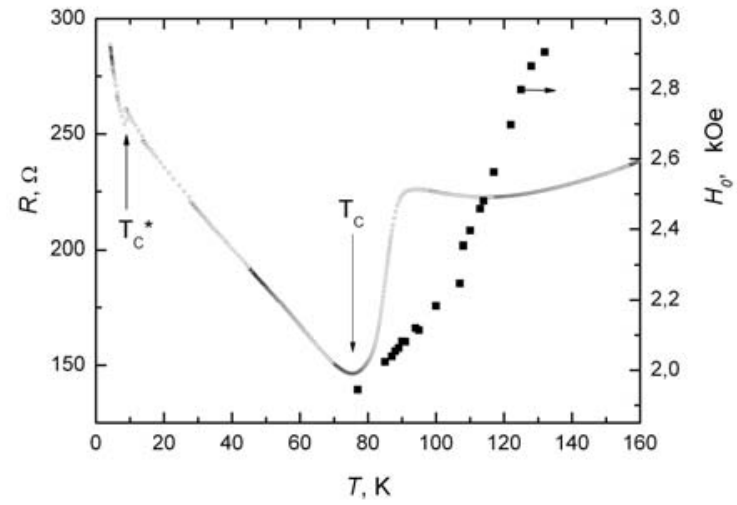

Fig.3. Temperature dependence of the resistance of mesastructure with area of $400 \mu \mathrm{m}^{2}$ and thickness $d_{M}=17 \mathrm{~nm}$ of the LMO interlayer. The superconducting transition temperature is $T_{C}=78 \mathrm{~K}$ for $\mathrm{YBCO}$ and $T_{C}{ }^{*}=8.7 \mathrm{~K}$ for $\mathrm{Nb}-\mathrm{Au}$. Squares show the temperature dependence of the ferromagnetic resonance field of $\mathrm{Au} / \mathrm{LMO} / \mathrm{YBCO}$ heterostructure with $d_{M}=16 \mathrm{~nm}$.

The differential resistance $R_{d}=\mathrm{d} V / \mathrm{d} I$ was measured separately using lock-in amplifier with the synchronous detector and the built-in modulating unit of signal generator. Modulating signal was applied to the mesastructure through the dc feed noise filtering circuit. Fig.3. shows temperature dependence of the resistance of mesa-structure along with the ferromagnetic resonance experimental function of $\mathrm{Au} / \mathrm{LMO} / \mathrm{YBCO}$ heterostructure, made without superconducting $\mathrm{Nb}$ film. The magnetic uniaxial anisotropy $H_{s}=375 \mathrm{Oe}$, and magnetization $M_{0}=1.75 \mu \mathrm{B} / \mathrm{Mn}$ were obtained.

The case when magnetic field was applied to mesastructure is shown in Fig.4. The temperature dependence of differential conductivity $G=1 / R_{d}$ is presented in Fig.4a when no magnetic field was applied. Fig.4b shows the $G(V)$ dependences under the action of a magnetic field. An asymmetry of $G(V)$ function for positive and negative biasing $V$ is seen, which could be caused by the shape of the tunnelling barrier and the difference of work functions of contacting to the LMO, the YBCO and Au films. An other feature is the change of $G(V)$ under the influence of magnetic field. This change of $G(V)$ occurs only within the voltage range at the mesa-structure up to the voltage of niobium energy gap. It could not be attributed to the Zeeman splitting of the density of states of the electrodes [13], since the applied magnetic field $H$ is much smaller than the Zeeman splitting field. After the transition to the ferromagnetic state of the LMO layer at temperature $T<$ $140 \mathrm{~K}$, a spin-polarized current appears in the mesastructure [3] and magnetic field affects the tunneling conductivity due to spin polarization [14]. The observed value of magnetoresistance of mesa-structure is typical for manganites [12] and took place only at temperatures below the critical temperature of bilayer $\mathrm{Au}-\mathrm{Nb}$. With an increase of dc bias voltage above $3 \mathrm{mV}$ (about twice higher than the niobium energy gap), the conductivity of the mesa-structure no longer depends on magnetic field [16]. The magnetoresistance measured at wider magnetic field sweeping range is shown in Fig.5. The hysteresis, typical for ferromagnets caused by the change in 
magnetic field direction takes place. Upon an increase of dc bias voltage up to $10 \mathrm{mV}$, the differential resistance of mesa-structure becomes independent of the magnetic field (curve 2 in Fig.5).
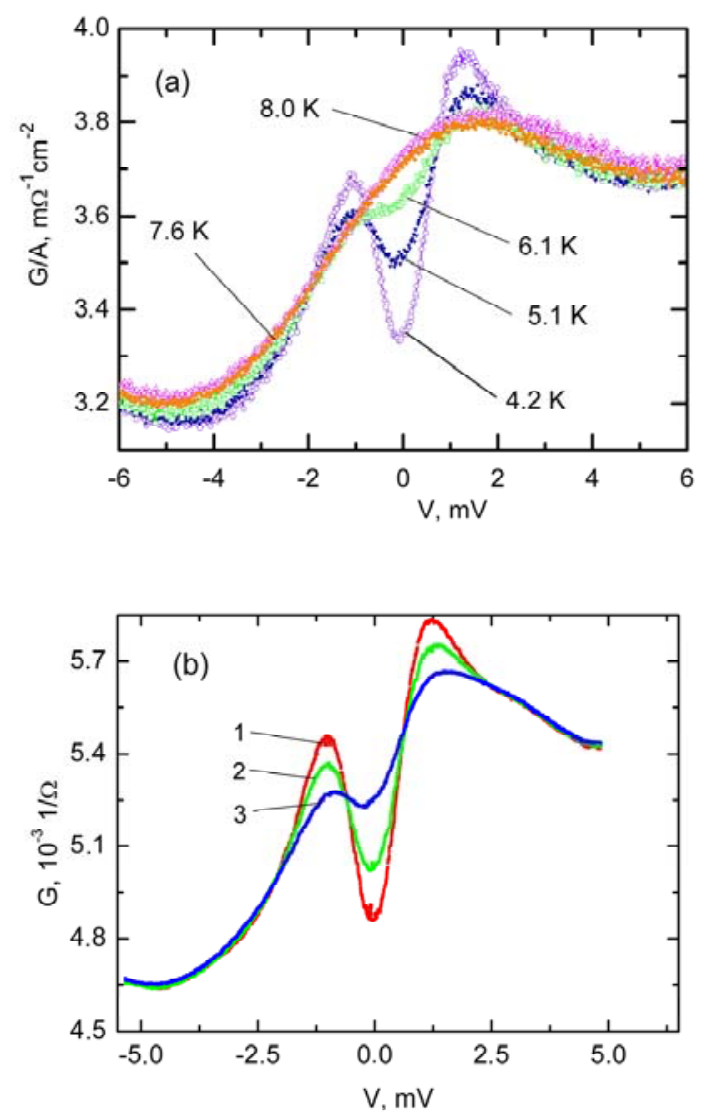

Fig.4. (color online) Family of differential conductivity $G$ vs. voltage $V$ for mesa-structure with $d_{M}=5.6 \mathrm{~nm}, A=140 \mu \mathrm{m}^{2}$. (a) Characteristic conductivity $G / A$ measured at fixed temperatures in the range $4.2-8 \mathrm{~K}$ for $H=0$; (b) for fixed magnetic field directed parallel to the substrate plane, $1-H=0$ Oe, $2-H=263$ Oe, $3-H=526$ Oe. Ambient temperature $T=4.2 \mathrm{~K}$.

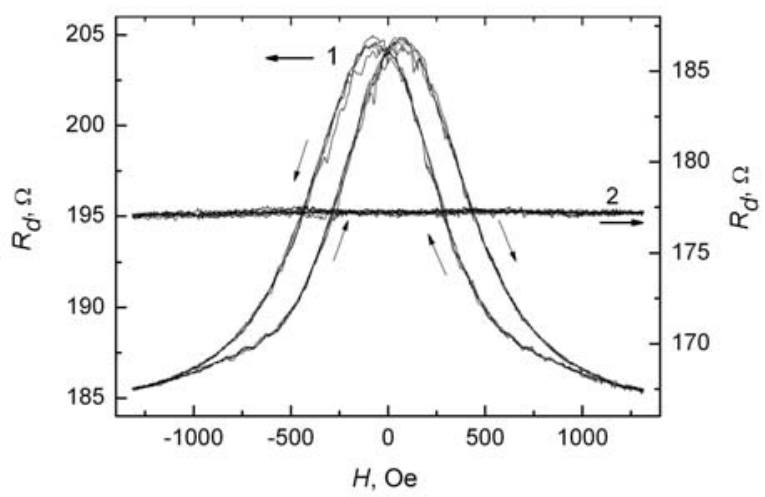

Fig.5. Magnetoresistance of mesa-structure with area of $20 \mathrm{x}$ $20 \mu \mathrm{m}^{2}$ at $T=4.2 \mathrm{~K}$ measured using a few runs of the external magnetic field applied in parallel to the plane of the substrate. Arrows indicate the direction of magnetic field change. Family of curves 1 was obtained for zero bias voltage $(V=0)$; family 2 was obtained for fixed $V=10 \mathrm{mV}$.
Results of microwave measurements are shown in Fig.6. Fig.6a demonstrates a family of $P(V)$ dependences of the microwave power emitted by mesa-structure vs. bias voltage, obtained for several values of the external magnetic field at $T=4.2 \mathrm{~K}$. As the temperature rises above the critical temperature of superconducting $\mathrm{Nb}-\mathrm{Au}$ bilayer, the microwave generation disappears. The smooth growth of $P$ with increasing $V$ is most likely caused by the shot noise of the mesa-structure. Symmetrically located relative to $V=0$ the excess signal in $P(V)$ dependence, significantly exceeding the noise level, are observed. At the microwave spectrum analyzer, the appearance of excess $P$ signal was manifested in the form of a sharp generation peak with a power of order of fraction of $\mathrm{pW}$ and with a linewidth of $\sim 50 \mathrm{MHz}$. The central frequency of the peak was tuned within the whole experimental frequency range $f=1-2$ GHz. Power $P$ of excess signal showed tendency to grow with increasing the bias current $I$ (or voltage $V$ ): the highest peaks were observed at larger voltages $V$. Note, that in the structures discussed in $[8,9]$, on the contrary, the generation frequency decreased with increasing bias current $I$. The dependence of the generation power $P$ on the magnetic field $H$ (for a fixed $V$ ) had an oscillating form with an average oscillation period of order 0.5 Oe. Fig.6a also indicates that an average range of bias voltages $\Delta V$ where microwave generation was observed is amounted of $\sim 8 \mathrm{mV}$. An estimate of the frequency/current coefficient of frequency tuning $\Delta f / \Delta I$ gives a value of $7.510^{12} \mathrm{~Hz} / \mathrm{A}$, where $\Delta I=\Delta V / R_{d}$. Note, $R_{d}$ was of order $60 \Omega$ and weakly depends on $V$. In our experiment the generation frequency $f$ and the corresponding voltage $V$ differs from the Josephson ratio 483.6 $\mathrm{GHz} / \mathrm{mV}$ by three orders in magnitude ( $\mathrm{h}$ is the Planck constant, e is the electron charge). Taking into account the absence of superconducting current, it is impossible to attribute such microwave generation to the non-stationary Josephson effect. Fig.6b shows the magnetic field dependence of the maximum values of the generation amplitudes $P$. Despite the fact that the amplitude of the detected signal is rugged and irregular in shape, the values of the maxima corresponding to both polarities of the bias voltage coincided well (see Fig. 6b). On one hand, the occurrence of microwave generation could be explained by the trivial mechanism of the coherent motion of magnetic vortices. However, on the other hand, the stability of the regular motion of vortices in superconducting structures is difficult to achieve, and it easily disturbed by external microwave influence [18]. An application to the mesa-structure of a monochromatic signal with a power up to $0.5 \mathrm{~mW}$ at a frequency $f=70 \mathrm{GHz}$ was resulted in a slightly (about $20 \%$ ) suppression the generation amplitude without changing in its shape. Moreover, the repeated measurements of dependences of $P(V)$ at fixed $H$ both with the external microwave signal of $70 \mathrm{GHz}$, and without it, completely coincided, repeating the ruggedness of $P(V)$. Consequently, neither the Josephson effect, nor the mechanism of coherent motion of magnetic vortices could explain the nature of the observed microwave generation. At the same time, it was 
theoretically shown [19] that the controlled rotation of spin vortices (skyrmions [20]) under the action of a constant spin-polarized current can excite stable narrowband microwave generation and, importantly, without the necessary condition for exceeding the threshold value of the electric current. Another coincidence of the results of the experiment and the model [19] is the increase of generation frequency with increasing of bias current through the mesa-structure. The strong spin-orbit interaction, which is relatively weak in the autonomous manganite LMO [21], and which is necessary for the formation of spin vortices, can be enhanced due to JahnTeller distortion in the mesa-structure due to the influence of the lower YBCO film grown on the NGO substrate [22], and also by the presence of an interlayer of gold, the spin-orbit interaction in which was analyzed in [23].
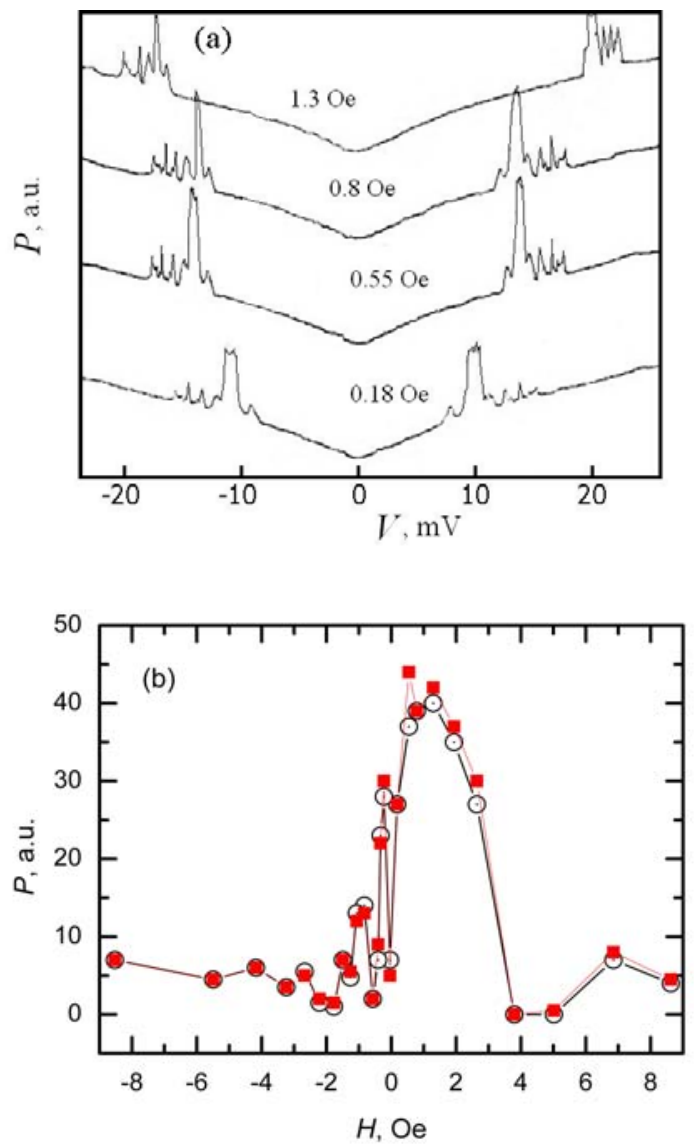

Fig.6. (a) The family of dependences of the signal $P$ on the bias voltage $V$ (shifted along the ordinate axis) obtained from the output of the quadratic detector at magnetic field changed in the range 0.18-1.3 Oe. Mesa-structure had the thickness of the LMO interlayer $d_{M}=5.6 \mathrm{~nm}$, the resistance $R=60 \Omega$, and size $A=1000 \mu \mathrm{m}^{2}$, (b) (color online) Dependence of the maximum microwave generation $P$ vs. magnetic field. Open circles denote peaks obtained with negative bias $V$, filled squares - at the positive bias.

In conclusion, the $\mathrm{YBCO} / \mathrm{LMO} / \mathrm{Au}-\mathrm{Nb}$ mesa-structure with the spin-filter properties demonstrated microwave generation in $\mathrm{GHz}$ frequency band with a linewidth of order $50 \mathrm{MHz}$ under the influence of external weak magnetic field $|H|<10$ Oe and the bias current. Generation frequency could be tuned by changing the bias current with a ratio of $\sim 10^{13} \mathrm{~Hz} / \mathrm{A}$, and its amplitude could be controlled by a weak magnetic field $H \sim 0.5$ Oe

The authors are grateful to I.V. Borisenko for a fruitful discussions of the results. The work was supported by the Russian Academy of Sciences, the RFBR project 16-29-14022 and the Scientific School NSh-8168.2016.2.

\section{References}

1. F. S. Bergeret, A. F. Volkov and K. B. Efetov, Rev. Mod. Phys., 77, 1321 (2005).

2. S. Kawabata, Y. Tanaka, A. A. Golubov, et al. J. Magn. Magn. Mater., 324, 3467 (2012).

3. R. Meservey, P.M. Tedrow, Phys. Rep. 238, 173 (1994).

4. T.S. Santos, J.S. Moodera, K.V. Raman, et al., Phys. Rev. Lett. 101, 147201 (2008).

5. E. Zhao, T. Löfwander, J.A. Sauls, Phys. Rev. B70, 134510 (2004).

6. J.C. Slonczewski, J. Magn. Magn. Mater.159, L1 (1996).

7. A. Manchon and S. Zhang, Phys. Rev. B79, 094422. (2009).

8. S.I. Kiselev, J.C. Sankey, I.N. Krivorotov, et al. Nature (London), 425, 380 (2003).

9. W.H. Rippard, M.R. Pufall, et al. Phys. Rev. Lett. 92, 027201. (2004).

10. V.S. Pribiag, I.N. Krivorotov, G.D. Fuchs, et al. Nature Physics, 3, 498 (2007).

11. Luqiao Liu, Chi-Feng Pai, et al., Phys. Rev. Lett., 109, 186602. (2012).

12. A.M. Petrzhik, G.A. Ovsyannikov, A.V. Shadrin, et al., JETP, 112, 1042. (2011).

13. J.S. Moodera, T.S. Santos, T. Nagahama, J. Phys. Cond. Matter. 19, 165202. (2007).

14. X. Hao, J.S. Moodera, R. Meservey, Phys. Rev. B42, 8235 (1990).

15. X. Renshaw Wang, C. J. Li,W. M. Lü, et al. Science, 349, Issue 6249, 717 (2015).

16. G.A. Ovsyannikov, Yu.V. Kislinskii, K.Y. Constantinian, JETP, 124, 628 (2017).

17. R. Monaco, M. Aaroe, J. Mygind, V.P. Koshelets, Phys. Rev. B79, 144521. (2009).

18. K.I. Konstantinyan, G.A. Ovsyannikov, L.E. Amatuni, Z.G. Ivanov, Sov. Phys. JETP, 72, 376 (1991).

19. F. Garcia-Sanchez, J Sampaio, et al., New J. Phys. 18, 075011 (2016).

20. A. Fert, V. Cros and J. Sampaio, Nature Nanotechnology, 8, 152 (2013).

21. M. Snamina, A.M. Ole's, Phys. Rev. B94, 214426 (2016).

22. I.V. Borisenko, M.A. Karpov, G. A. Ovsyannikov, Tech. Phys. Lett. 39, 1027 (2013).

23. A. Dal Corso, A. M. Conte, Phys. Rev. B75, 115106 (2005) 